\title{
Mobile crowd sensing for smart urban mobility
}

\author{
Dragan Stojanovic*, Bratislav Predic and \\ Natalija Stojanovic \\ University of Nis, Faculty of Electronic Engineering \\ Aleksandra Medvedeva 14, 18000 Nis, Serbia \\ *dragan.stojanovic@elfak.ni.ac.rs
}

\begin{abstract}
In this paper, we present the application of the mobile crowd-sensing paradigm in supporting efficient, safe and green mobility in urban environments. We have developed the CitySensing framework demonstrating the viability of a common crowd-sourcing platform applied to various urban mobility domains. We argue that today's mobile devices, with integrated or add-on sensors, can be efficiently used to crowd source diverse information in domains that are relevant to urban life and mobility (traffic, air quality and citizens' everyday activities). This is illustrated by three distinct mobile applications, developed on top of the CitySensing framework, that contribute to a common goal of smarter urban mobility. Commonly integrated accelerometer and GPS are used to infer traffic events and conditions. Externally attached or integrated air quality sensors enable suggestions for city areas adequate for outdoor activities on a specific day of the week or hour of the day. Mobile phone usage statistics and analysis can present valuable information to urban planning services to better adapt to citizens' habits and mobility. The analysis of this massive amount of crowd sensed data (so-called Big Data) within the cluster/cloud infrastructure enables detection of situations and events that influence human mobility, and dissemination of notifications and recommended actions.
\end{abstract}

\section{How to cite this book chapter:}

Stojanovic, D, Predic, B and Stojanovic, N. 2016. Mobile crowd sensing for smart urban mobility. In: Capineri, C, Haklay, M, Huang, H, Antoniou, V, Kettunen, J, Ostermann, F and Purves, R. (eds.) European Handbook of Crowdsourced Geographic Information, Pp. 371-382. London: Ubiquity Press. DOI: http://dx.doi. org/10.5334/bax.aa. License: CC-BY 4.0. 


\section{Keywords}

crowd sourcing, mobile sensing, smart city, smart mobility

\section{Introduction}

With advancements and the proliferation of mobile devices with increasing computing, communication and sensing capabilities, mobile users have become important sources of sensing data in a globally spread wireless sensor network (Campbell et al. 2008). Mobile crowd sensing represents an approach for a new sensing and geo-crowdsourcing paradigm that leverages the power of various mobile devices, such as smartphones, tablets, wearable devices, smart sensors, etc. It is based on the human ability to acquire local geospatial information and knowledge through sensor-enhanced mobile devices, and the possibility to share this information/knowledge with other users and a wide community (Goodchild 2007; Kamel Boulos et al. 2011). As the world population becomes increasingly urban, cities worldwide need to leverage information and communications technologies to improve their functions, enhance efficiency, improve competitiveness and the economy and provide better environment for their citizens; to become 'smarter'. Smart mobility is one of the key characteristics of a smart city, and also one of the biggest challenges that smart cities face (Batty et al. 2012). The world population makes more than $64 \%$ of all travel kilometers within urban environments, which is expected to triple by 2050 . Thus, a high priority for cities around the world is to support citizens' mobility within the urban environment based on safety, efficiency and environmental protection (Motta et al. 2015). Mobile crowd sensing paradigm will enable key methods, techniques and systems in that direction. The application of analysis, reasoning and data mining techniques on the mobile crowd sensed data provides useful insights in citizens' mobility and supports better citizen involvement in monitoring urban spaces.

In this article, we describe main concepts, methods and technologies of mobile crowd sensing in smart cities and present the CitySensing framework developed to support smart urban mobility through participation and intelligence of the crowd (Section 2). Several mobile demo applications have been developed based on this framework that illustrate the use of mobile crowd sensing in different city's scenarios and provide insights how such applications could improve citizens' mobility (Section 3). Although there are still open challenges and real-life deployments of developed applications, the initial evaluations look promising in supporting smart citizens' mobility, as concluded in Section 4.

\section{Mobile crowd sensing for smart cities}

The mobile crowd sensing refers to geo-crowdsourcing and VGI (Volunteered Geographic Information) paradigm in which mobile users use their mobile 
computing, communication and sensing devices to collect, locally process and analyze, as well as distribute geo-referenced information (Chatzimilioudis et al. 2012). Mobile crowd sensing paradigms have become the significant source of VGI and crowdsourced geo-information owing to the large number of mobile devices carried by people worldwide to support their daily activities (Zhang et al. 2014). Such crowd sensed data is filtered, aggregated, processed and analyzed at the server(s) and appropriate information services and notifications are delivered to mobile users to support their mobility. In such scenario mobile users are both the producers of mobility sensing data, and the consumers of services, notifications and recommendations based on processing and analysis of the massive amount of crowd sensed data at server(s).

There are several sources of mobility sensing data originated at mobile devices, classified as:

- Physical sensors,

- Virtual (logical) sensors,

- Social sensors.

Physical sensors include sensors integrated in, or attached to mobile devices (smart phones, tablets, etc.), such as: GPS, microphone, camera, ambient light sensor, accelerometer, gyroscope, compass, proximity sensor and also the temperature and humidity sensors available on advanced smartphones. The development of wearable and pervasive systems, such as Sensordrone ${ }^{1}$ and iWatch ${ }^{2}$, provides integration of additional sophisticated sensors, worn by users and attached to their mobile devices, to measure air pollution, personal health parameters and the emotional and physiological status of users. Virtual sensors are not hardware sensors but software applications that run at user devices and collect information about users, their profile and preferences, detecting their context and situation. Such sensors detect information related to user communications (voice, SMS, etc.), user activities and interaction with devices (active applications, application in focus, the type of the interaction, etc.), user preferences and profile, user-generated content (texts, speech, videos, photos, sounds), etc. Virtually sensed information is referenced in space and time and attached to a certain location, symbolic or geographic. Social sensors detect user social status and activities, social network and social media interactions (tags, likes, Tweets, photos, etc.), currently connected friends and their status, connections in vicinity, etc. Some of such information can be detected by accessing social network/media services through appropriate APIs.

Mobile crowd sensed data collection can be performed with the participation and active user involvement, called participatory sensing, or without active involvement of users, i.e. opportunistic sensing (Campbell et al. 2008). The

\footnotetext{
${ }^{1} \mathrm{http}: / /$ sensorcon.com/products/sensordrone-multisensor-tool

${ }^{2}$ https://www.apple.com/watch/
} 
research of mobile crowd sensing for smart cities focuses on the collection, representation, filtering, aggregation, processing and analysis of large volumes of mobility sensing data, both on mobile devices and at central server(s). Such data describe citizens' movements and activities, environmental conditions they face (e.g. high pollution level, traffic congestion, crowded or noisy surroundings), communication and interaction they make (talking, searching on the Web, tagging a photo, connecting with friends, etc.). The analysis and mining of mobility data obtained through mobile crowd sensing provide insights into important features of the urban mobility, behavior of people moving around a city and possible prediction of future mobility. There are two approaches for collection and distribution of mobile crowd sensed data:

- Server-based approach - Sensed data in its raw form are sent to a server without processing and analysis at the mobile devices. Such an approach results in very high demands for wireless network bandwidth, as well as for storage, processing and analysis of raw mobility data at the server.

- Distributed approach - Data from physical, virtual, and social sensors are collected, processed and analyzed at the mobile devices and high level contextual and mobility information are sent to a server.

Mobile crowd sensed data and information represent the foundation for mobility information services that are delivered to the same users to support their smarter mobility (Ilarri et al. 2015). The crowd sensed data collected from a large number of mobile users/moving objects are characterized by massive volume, velocity and variety, and can be regarded as Big Data. The server applications running on a cluster/cloud that support mobile crowd sensing should provide data collection, processing, reasoning, analysis and mining over massive mobility data sets, from structured and unstructured sources. Such a collaborative crowd intelligence provides detection of aggregated mobility patterns and trajectories, group activities and behavior, as well as complex situations (e.g. interesting places, traffic congestions, popular city routes or crowded evacuation paths in an emergency situation) in smart cities (Cardone et al. 2013).

We have developed a framework, named CitySensing, to support the development of mobile crowd sensing applications for various smart city scenarios. The CitySensing framework consists of mobile application components, server components and visualization/analytics components organized in a distributed architecture given in Figure 1. It supports both opportunistic and participatory methods of crowd sensed data collection using various physical, virtual and social sensors available in today's mobile devices. It fully leverages processing, sensing and communication capabilities of mobile devices and provides distributed and scalable storage, processing, analysis and mining of crowd sensed mobility data at mobile devices within CitySensing mobile components. High-level mobility information generated at users' devices is further aggregated, processed and analyzed at the CitySensing server components running 


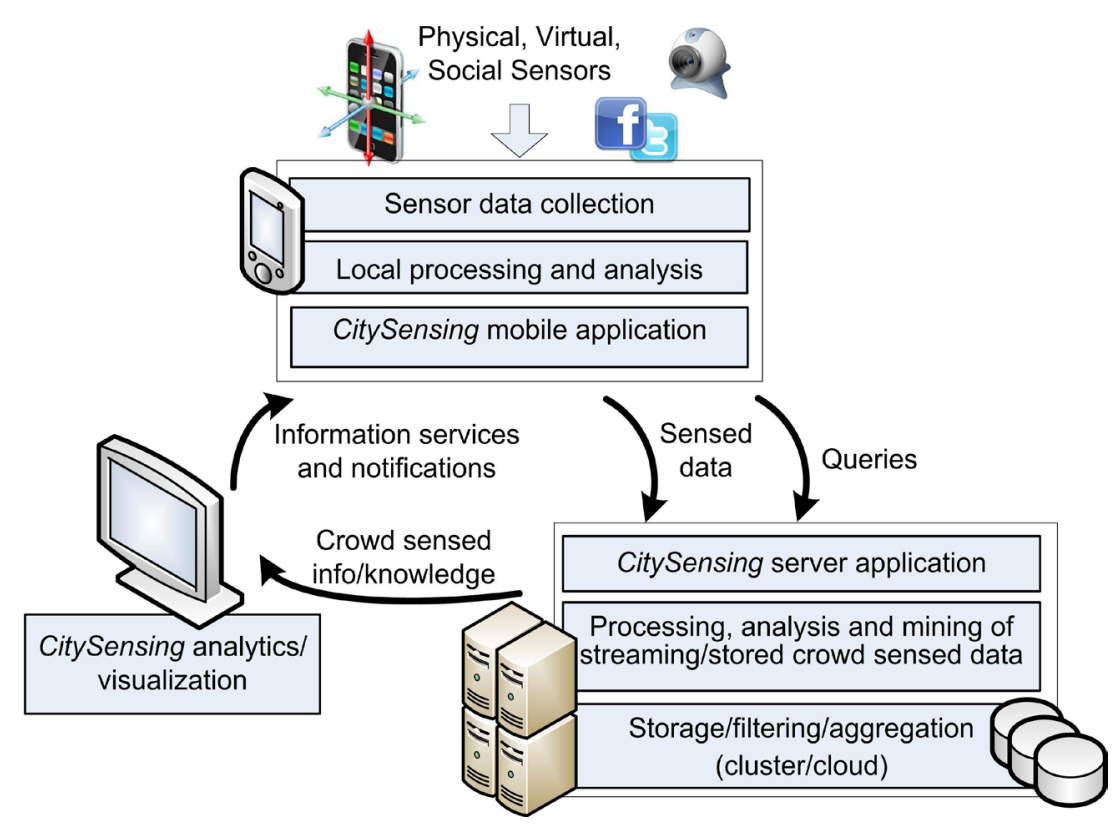

Figure 1: A general architecture of CitySensing framework.

on a cluster/cloud infrastructure. The CitySensing framework is based on the open-source sensor data collection framework, Funf, ${ }^{3}$ and the data analysis and mining framework WEKA ${ }^{4}$. For processing and analysis of big spatio-temporal data, CitySensing server components are based on distributed processing frameworks, such as MapReduce/Hadoop ${ }^{5}$ and Spark, ${ }^{6}$ for processing offline crowd-sensed data stored in a distributed file system over a cloud/cluster. For real-time processing and analysis of large crowd sensed data streams appropriate data stream processing frameworks are employed, such as Apache Storm ${ }^{7}$ and Spark Streaming.

\section{Dynamic crowd-sensed information for smart mobility}

Smart mobility in urban environments is based on integrated mobile information services to support efficient, safe and green transport of people and goods and user activities in smart cities. We are developing several demo smart

\footnotetext{
${ }^{3}$ http://www.funf.org/

${ }^{4}$ http://www.cs.waikato.ac.nz/ml/weka/

5 https://hadoop.apache.org/

${ }^{6}$ https://spark.apache.org/

${ }^{7}$ https://storm.apache.org/
} 
mobility applications, namely DriveSense, ExposureSense and UrbanSense, based on CitySensing framework, to support different urban mobility scenarios.

\section{Crowd-sensing traffic information}

Recent research on smart mobility has started to leverage the principle of mobile crowd sensing by employing drivers/passengers and pedestrians equipped with mobile devices as real-time sources of navigation, environmental and traffic information. The basic idea implemented in DriveSense application is to use drivers/passengers and pedestrians as moving sensor platforms that can collect traffic, driver and road related conditions, events and information and send them to a central server, or to mobile devices in the vicinity, using appropriate wireless communication mechanism (3G, Wi-Fi, Bluetooth, etc.). Such dynamic information should support citizens in their everyday mobility, both indoors and outdoors.

Regarding vehicle transport, mobile crowd sensing systems could detect and report traffic events and conditions, the state of road infrastructure, driver behavior and activities, as well as accidental events:

- Traffic events and condition

- Travel times over the street segments.

- Slowed down traffic (traffic jams).

- Congestion points (start-stop locations)

- Parking places.

- Traffic stops.

- Road infrastructure state

- Bumpy road.

- Slippery road surface.

- Damaged road surface location (potholes).

- Dynamic traffic events and driver behavior (accidents and situations that could potentially cause accidents)

- Sudden breaking, decelerations and acceleration.

- Lateral skidding.

- Violent (sudden) change of direction and traffic lane at a high speed.

- A vehicle moved out of a road; an accident or a collision happened, etc.

The DriveSense application is used as a tool for crowd sensing of dynamic traffic information, and also as dynamic navigation service based on current traffic conditions. Both participatory and opportunistic modes can be used for collection of information on traffic condition and events (Figure 2a). During driving, relevant traffic events (e.g. sudden lane changes, numerous hard breaking events, etc.) are reported by moving users and delivered to all drivers in affected street segments. As a result, a service also proposes re-routing to drivers to avoid an intersection that is probably heavily congested, or potentially unsafe for driving. Spatio-temporal aggregation and analysis of crowd sensed traffic information 


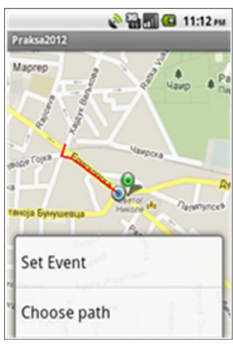

a)

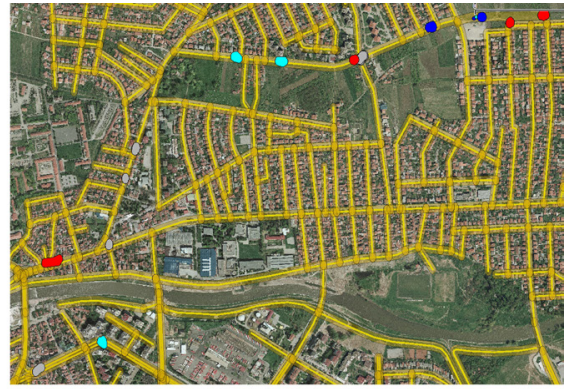

b)

Figure 2: DriveSense application a) Mobility information service b) Space-time clustering of reported traffic events.

collected over longer time periods can provide valuable information to city traffic service and identify parts of city's street network that could affect safe and efficient traffic. The results of this analysis are shown in Figure $2 b$ showing parts of a street network with detected potholes (blue dots), areas with excessive harsh breaking (red dots) and frequent violent lane changes (green dots).

\section{Air quality sensing and reporting}

Air pollution is identified as a major health concern in urban areas. The citizens are also exposed to other types of urban 'pollutions', such as noise and electromagnetic waves. By applying crowd sensing concepts to users with mobile devices, both citizens and city authorities can detect and be aware of pollution exposure during their everyday activities in urban areas, especially for pedestrians, as demonstrated by ExposureSense application (Figure 3). As with previous DriveSense example, users of ExposureSense act as both sources of information on air quality and consumers of such information which support planning their daily physical activities in city areas with less exposure to pollution. By cross-correlating their physical activities with pollution information, users can receive information and services regarding their pollution exposure and recommendation for activities, as well as navigation instructions to 'cleaner' areas (Predic et al. 2013).

The ExposureSense application can act as a personal diary of physical activities giving both map view (Figure $3 \mathrm{a}$ ) and timeline view (Figure $3 \mathrm{~b}$ ). This information can be combined with sensed air-quality parameters (like temperature, concentration of different particles or gasses in the air). Figure 3c shows map visualization of $\mathrm{NO}_{2}$ concentration sensed using sensors attached to a mobile device during typical recreation activity. Summarized information is shown in a calendar type view in Figure 3d.

The drivers/passengers and pedestrians are also the consumers of smart mobility information services generated by such crowd intelligence approach. 


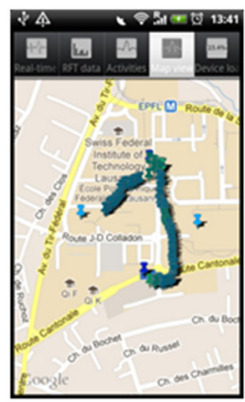

a)

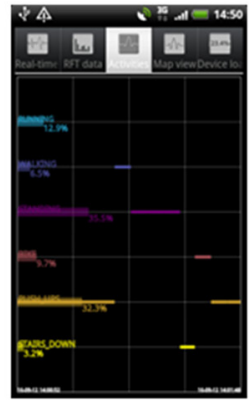

b)

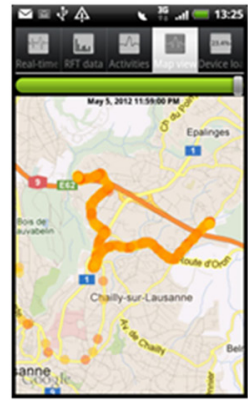

c)

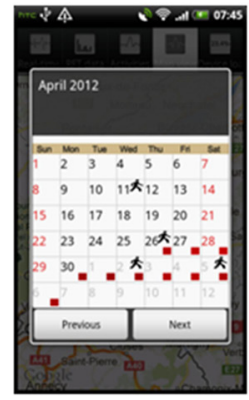

d)

Figure 3: ExposureSense mobile crowd sensing application a) Map view of activities b) Timeline view of activities c) Exposure to $\mathrm{NO}_{2}$ during recreation activity, d) Summary information of activities and exposures to pollutants.

They receive real-time traffic information, navigation instructions and notifications, recommended activities and environmental conditions and actuate upon them (Predic \& Stojanovic 2015). City authorities can use such crowd intelligence to improve urban mobility by detecting mobility behaviors and patterns of citizens/tourists movement. Such behavior and movement patterns, related to background geographic information (POI, road network data, indoor maps), time context (time of the day, day of the week, month, season), weather conditions, means of transport, environmental conditions, social/cultural events in the city or indoors, can enable better understanding of the city dynamics.

\section{Behavior patterns sensing using mobile activity collection}

Ubiquitous and transparent collection of mobile usage statistics as geo-referenced mobility data can provide valuable information to urban planning city services. Crowd sensed information showing urban areas where mobile users tend to spend their leisure/break time, types of physical activities during parts of a day, and their physical environment during these activities can allow city planners to adapt city's business and recreational areas according to citizens' mobility in urban environment. The UrbanSense mobile application has been developed as a mobile diary service that detects and stores information about users' environment and mobile interaction by collecting following data:

- Physical environment: temperature, air pressure, air humidity, light intensity, etc.

- Phone communication usage: SMS sending/receiving and phone calls.

- User's physical activities.

- Wireless communication devices in surrounding, e.g. WiFi access points, cellular network base stations, Bluetooth devices, other mobile devices, etc. 
- Active usage of a phone based on a screen on/off state.

- Usage of certain types of mobile applications especially social networking ones.

- Recording of photos/videos using phone cameras.

- Playback of sound/video files.

- Battery level / charging data.

The UrbanSense demo application consists of a mobile service tasked with collection of sensing data, a mobile application used for service configuration and access to mobility information services, as well as a server(s) which receives, stores, aggregates, processes and analyses crowd sensed data. The mobile service collects data in a local mobile database in predefined time intervals (usually daily) and sends the collected data to the server. A service configuration allows the user to filter which types of activity information can be collected during specific periods of a day/week, for the reason of privacy preservation (Figure 4a). All sensitive personal information in collected data is hashed in order to preserve data topology and semantics while maintaining anonymity and privacy. This includes phone numbers and SMS messages' content. Prior to submitting collected data to the server user can view and filter it in the mobile application, as shown in Figure $4 \mathrm{~b}$. At the server, collected data are processed and analyzed by applying spatio-temporal clustering techniques and social connections based on SMS and voice calls history are detected. After clustering, urban areas with intensive physical and communication activities can be visualized on a map and filtered according to the type and the period of day/week (Figure 4c).

The main goal of UrbanSense application is to infer social network connections and detect urban areas with intensive social interaction, or which contain a specific mobile usage pattern. The identification of such areas, along with time periods when certain patterns of social activities occurred, can be used for urban planning, coverage of city areas with marketing, sports or leisure events and supporting infrastructure.

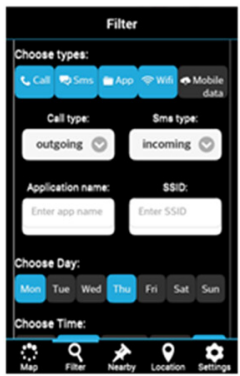

a)

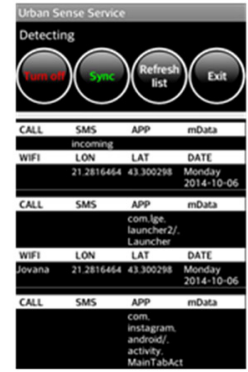

b)
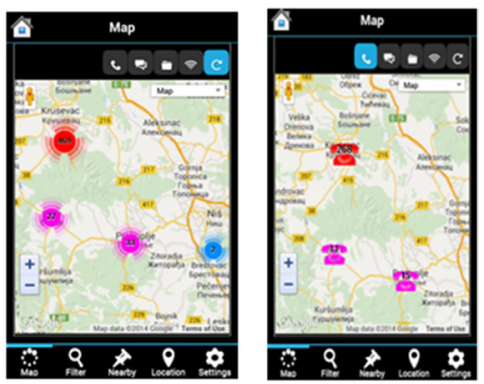

c)

Figure 4: UrbanSense application a) Data collection configuration b) Overview and filtering of collected data c) Spatio-temporal clustering of collected communication data. 


\section{Server-side processing techniques for crowd-sourced spatial data}

Using their mobile devices while walking and driving, and participating in social networks and media, citizens provide a wealth of information related to their mobility in urban environments and can support numerous applications for the benefit of the urban community. Smart mobility crowd sensing applications presented so far are based on local processing and analysis of mobile sensor data to detect high level, semantic mobility information at mobile devices. Such information is transferred to central server(s) that perform aggregation of semantic mobility data collected from a large number of mobile users and provide query processing, reasoning, analysis and mining, over big mobility data sets. The server performs detection of aggregated mobility patterns and trajectories, collective activities and behavior, as well as complex mobility situations (e.g. traffic congestions, risky and dangerous events, frequent city routes, crowded evacuation paths in an emergency situation, popular places and orders of their visit, etc.) (Ilarri et al. 2015). Mobile crowd sensed data processing, analysis and visualization, either for single citizen or aggregated set of citizens through time, enables early detection of situations and events that affect urban mobility, as well as the estimation of their impact to the community. Also, they provide dissemination of proactive location-based and context-aware services, notifications and alert messages, as well as recommendations for the actions to citizens on the move.

Crowd sensed data collected from UrbanSense mobile application can be analyzed and visualized to detect user activities on the move. The timeline and a map overlay visualization of per-user activity data collected by UrbanSense application is shown in Figure $5 \mathrm{a}$.

Crowd sensed data from numerous mobile citizens are streamed to server applications based on big data processing and analysis frameworks deployed over distributed computing infrastructure, such as MapReduce/Hadoop, Apache Spark and Apache Storm and Web-based visual analytics components and technologies. Such architecture allows building of flexible and scalable crowd-sourcing software services that can effectively analyze citizens' mobility patterns in modern cities. In Figure $5 b$, a heavy traffic on major city streets (different levels of red color) detected by crowd-sourcing traffic information is co-occurred with bursts of 'traffic' on Twitter around the same locations (blue colored circles). The contents of Tweets could offer a clear and fast explanation of the traffic jam, e.g. a protest of local farmers.

\section{Conclusions}

Presented demo mobile applications confirm that mobile crowd sensing represents a very promising approach for mobilization of citizens in order to improve and adapt their urban mobility. Demonstrated and other freely available mobile crowd sensing applications suggest that crowd-sourcing dynamic 

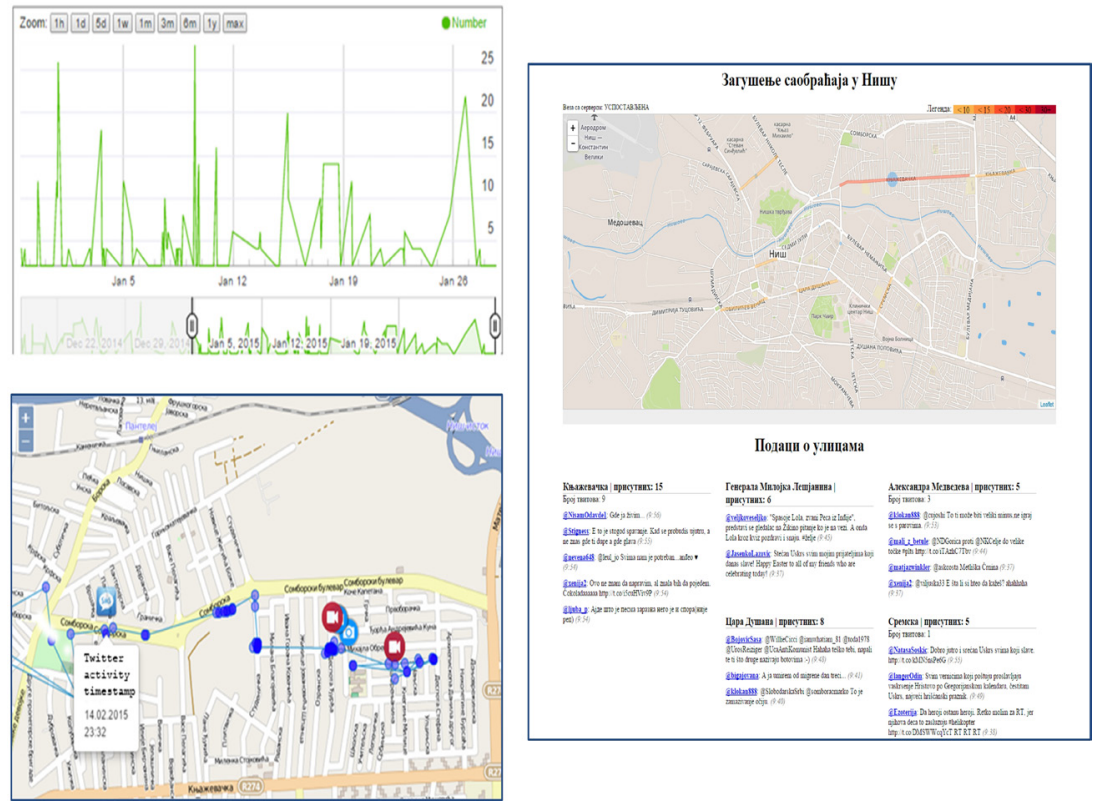

a)

b)

Figure 5: Visualization and analysis of crowd sensed mobility data a) Citizen's activities visualized on a timeline and a city map b) Traffic congestion and Twitter activities in Nis based on Storm server application.

geographic information has potential to improve various aspects of urban life and urban mobility. In this paper we focused on probably the most prominent domains such as traffic, air quality and citizens' activities on the move that have the great impact on citizens' mobility and city dynamics. Presented research work demonstrates that other aspects of urban life and planning can also benefit from crowd-sourcing geographic information.

There are still a number of open issues and research challenges in mobile crowd sensing approach applied to smart cities. To ensure the citizens' participation, reliability, security and privacy must be covered in the future work. Approproate methods and tools should be investigated and developed to ensure the accuracy of the information collected, an adequate level of privacy to citizens, as well as citizens motivation through gamification and micro payments. Also, the future research must take into account the high volume, velocity, variety and veracity of data provided by the citizens, and collected from other structured and unstructured data sources (e.g. sensor networks, Internet of Things, etc.). Accordingly, advanced methods and techniques for processing, analysis and visualization of such Big mobility data should be developed, that will unearth new information and knowledge by exploring the correlation and 
patterns across multi-sources of crowd sensed data. It will provide more effective services and solutions for citizens and decision makers in urban mobility.

\section{References}

Batty, M., Axhausen, K. W., Giannotti, F., Pozdnoukhov, A., Bazzani, A., Wachowicz, M., Ouzounis, G., \& Portugali, Y. 2012. Smart cities of the future. European Physical Journal: Special Topics, 214: 481-518. DOI: http:// dx.doi.org/10.1140/epjst/e2012-01703-3

Campbell, A. T., Eisenman, S. B., Lane, N. D., Miluzzo, E., Peterson, R. A., Lu, H., Zheng, X., Musolesi, M., Fodor, K., \& Ahn, G.-S. 2008. The Rise of People-Centric Sensing. IEEE Internet Computing, 12(4): 12-21. DOI: http:// dx.doi.org/10.1109/MIC.2008.90

Cardone, G., Foschini, L., Bellavista, P., Corradi, A., Borcea, C., Talasila, M., \& Curtmola R. 2013. Fostering participaction in smart cities: A geo-social crowdsensing platform. IEEE Communications Magazine, 51(6): 112-119. DOI: http://dx.doi.org/10.1109/MCOM.2013.6525603

Chatzimilioudis, G., Konstantinidis, A., Laoudias, C., \& Zeinalipour-Yazti, D. 2012. Crowdsourcing with Smartphones. IEEE Internet Computing, 16(5): 36-44. DOI: http://dx.doi.org/10.1109/MIC.2012.70

Goodchild, M. F. 2007. Citizens as sensors: the world of volunteered geography. GeoJournal, 69(4):211-221. DOI: http://dx.doi.org/10.1007/s10708-007-9111-y

Ilarri, S., Stojanovic, D., \& Ray, C. 2015. Semantic management of moving objects: A vision towards smart mobility. Expert Systems with Applications, 42(3): 1418-1435. DOI: http://dx.doi.org/10.1016/j.eswa.2014.08.057

Kamel Boulos, M. N., Resch, B., Crowley, D. N., Breslin, J. G., Sohn, G., Burtner, R., Pike, W. A., Jezierski, E., \& Chuang, K-Y. 2011. Crowdsourcing, citizen sensing and sensor web technologies for public and environmental health surveillance and crisis management: trends, OGC standards and application examples. International Journal of Health Geographics, 10(67): 1-29. DOI: http://dx.doi.org/10.1186/1476-072X-10-67

Motta, G., Sacco, D., Ma, T., You, L., \& Liu, K. 2015. Personal Mobility Service System in Urban Areas : the IRMA Project. In: IEEE Symposium on ServiceOriented System Engineering, pp. 1-10.

Predic, B., \& Stojanovic, D. 2015. Enhancing driver situational awareness through crowd intelligence. Expert Systems with Applications, 42(11): 48924909. DOI: http://dx.doi.org/10.1016/j.eswa.2015.02.013

Predic, B., Yan, Z., Eberle, J., Stojanovic, D., \& Aberer, K. 2013. ExposureSense: Integrating daily activities with air quality using mobile participatory sensing. In: 2013 IEEE International Conference on Pervasive Computing and Communications Workshops (PERCOM Workshops), pp. 303-305.

Zhang, D., Wang, L., Xiong, H., \& Guo, B. 2014. 4W1H in Mobile Crowd Sensing. Communications Magazine, IEEE, 52(8): 42-48. DOI: http://dx.doi. org/10.1109/MCOM.2014.6871668 